\title{
Acceleration of slip motion in deep extensions of seismogenic faults in and below the seismogenic region
}

\author{
Bruce E. Hobbs ${ }^{1}$, Hidemi Tanaka², and Yoshihisa $\mathrm{Iio}^{3}$ \\ ${ }^{1}$ CSIRO, Perth 6102, Australia \\ ${ }^{2}$ Department of Earth and Planetary Science, The University of Tokyo, Tokyo, Japan \\ ${ }^{3}$ Earthquake Research Institute, The University of Tokyo, Tokyo, Japan
}

(Received April 16, 2002; Revised September 30, 2002; Accepted September 30, 2002)

\begin{abstract}
This paper addresses concepts presented in Session 7 of the conference on "Slip and Flow Processes Near the Base of the Seismogenic Zone" held at Sendai, Japan in November, 2001. The questions raised in this session were associated with the downward extensions of seismic faults into the lower crust. The important issue is whether asiesmic slip accelerates on downward extensions prior to large earthquakes on the upper, seismic part. If this is the case, then such movement may be measurable as a precursor to large seismic events as accelerated tilt and/or distortion at the surface. Associated issues involve the geometry of downward extensions of faults, the mechanisms of localisation in the lower crust, and the mechanisms for earthquake generation near the base of the upper crust. The outcomes from this session are that aseismic slip in the lower crust could be generated by several mechanisms of localisation including yield of an elastic-viscous-plastic material, softening (including, in particular, thermal softening) of an elastic-viscous material and ductile fracture. Fine scale modelling of localisation in the lower crust is still required to resolve the issue whether accelerated motion precedes seismic slip in the upper crust. Such modelling also demands a better understanding of crustal rheology than we have at present.
\end{abstract}

\section{Introduction}

This paper is a discussion of the concepts presented in papers given in Session 7 of the conference on "Slip and Flow Processes Near the Base of the Seismogenic Zone" held at Sendai, Japan from November 5 to 8, 2001.

The important questions discussed in that session included:

- Under what conditions is deformation localized in the lower crust and, what is the mechanism for localisation?

- What is the geometry of the localised downward extensions of seismogenic faults into the lower crust? Are such extensions more or less parallel to, or can they be at a high angle to, seismogenic faults in the upper crust?

- What mechanisms are involved in the faulting process associated with large earthquakes near the base of the seismogenic zone?

- Does aseismic slip on the downward extensions of faults accelerate before large intraplate earthquakes occur? If this is the case, then this process implies an important, measurable precursor to large earthquakes.

First, some definitions are pertinent. This arises for two reasons. Firstly, communication is difficult between seismologists for whom "long term" means 100 's of years and geologists for whom $10^{6}$ years can be a short time scale. Secondly,

Copy right (C) The Society of Geomagnetism and Earth, Planetary and Space Sciences (SGEPSS); The Seismological Society of Japan; The Volcanological Society of Japan; The Geodetic Society of Japan; The Japanese Society for Planetary Sciences. the session concentrated on constitutive relations more complicated than the classical Byerlee Law, and non-linear viscous constitutive relations commonly used in the literature. As will be discussed below, such classical relations do not seem to be adequate, by themselves, to explain the observations. A range of relations were discussed in this session including (i) thermal-viscous coupling, (ii) ductile fracture, (iii) combinations of rate and state variable friction with exponential and power law creep, and (iv) frictional behaviour involving a combination of viscous and state variable mechanisms.

Throughout this paper we use the terms elastic, viscous, and plastic to mean the following: Elastic pertains to thermally and pressure insensitive, recoverable deformation. All materials have an element of elastic behaviour. Plastic pertains to pressure sensitive and thermally insensitive, permanent deformation characterised by a yield condition. The strong pressure sensitivity of plastic constitutive behaviour implies frictional behaviour, with or without dilatancy, at the micro-void and/or micro-crack scale and/or at the large joint or fault scale. Mohr-Coulomb behaviour (Vermeer and de Borst, 1984) is a special example of a plastic material and Byerlee behaviour (Goetze and Evans, 1979) is identical to Amonton behaviour (Backofen, 1972), if failure occurs on a single surface, or is a special example of Mohr-Coulomb behaviour with cohesion equal to zero if failure occurs in a continuous or localised manner through the bulk of the material. Viscous pertains to pressure insensitive, thermally sensitive, permanent deformation characterised by no yield condition. The classical linear and non-linear "Power Law Creep" 
materials are special cases of viscous materials undergoing steady state flow. Such usage is consistent with that used in the mechanics literature although the terms are used extremely loosely in the geological/geophysical literature.

Although it is possible to place fairly precise definitions on terms such as brittle, ductile, viscous and plastic, it is becoming apparent, as people attempt to quantitatively model the behaviour of the lithosphere, that real rocks have constitutive properties that are combinations of all of these "pure" behaviours. Thus it has long been known (for example see Ashby and Jones, 1986) that the tensile strength of ceramic like materials such as rocks is strain-rate dependent and hence probably temperature dependent. This means that even purely brittle materials display elements of viscous behaviour. An example here is the realisation that the brittle frictional behaviour of sliding rock surfaces at low temperatures and pressures can be described by a dependence on the sliding velocity which involves thermally activated terms (see Rice et al., 2001, for a synthesis). This "composite" constitutive behaviour of rocks seems to be emerging as an important issue and occupied a considerable part of the proceedings at the Sendai Conference.

Note that the terms brittle and ductile refer to the physical characteristics of deformation. That is, brittle refers to a solid that fractures early in the deformation history whereas ductile refers to a solid that undergoes considerable flow before fracture develops. On the other hand, terms such as plastic and viscous refer to constitutive behaviour as outlined above. A plastic material may be brittle or ductile depending on the conditions of deformation; a plastic material may localise even though it is strain hardening and displays high ductility. A viscous material is commonly highly ductile but it can localise if a softening process (damage, chemical weakening, thermal softening) occurs. There are many examples in the literature where localisation is taken to mean brittle behaviour; this is not necessarily the case. We want to emphasise that there is not a one to one correspondence between the physical character of deformation and the pertinent constitutive properties.

The crust of the Earth is commonly divided into the Upper Crust with dominantly "brittle" behaviour and the Lower Crust with dominantly "ductile" behaviour. Under the terminology used here, these two regions would commonly be characterised by elastic-plastic behaviour and elastic-viscous (+plastic?) constitutive behaviour. It is important to note the specific definition of "the seismogenic zone" accepted by the Sendai Conference, namely: that part of the upper crust of the Earth where large earthquakes occur. Thus, this definition is independent of the specific mechanisms of earthquake production and is independent of the specific constitutive behaviour of the crust. In particular, the definition does not refer specifically to the "brittle-ductile transition" as a definition of the base of the seismogenic region. Such a divorce between observations of seismicity and hypotheses concerning constitutive behaviour is fundamental. It opens the opportunity to consider a range of constitutive behaviour not traditionally employed including viscous-plastic and thermoviscous relationships.

As a prelude to concepts discussed in this paper a number of mechanisms (involving both plastic and viscous be- haviour) for the production of large earthquakes at or near the base of the seismogenic zone were discussed at the Sendai Conference:

- The classical mechanism involving instability of frictional sliding in the brittle regime (Byerlee Friction) as considered by a number of authors including Byerlee (1967), Dieterich (1979, 1981), Ruina (1983), and Gu et al. (1984).

- Brittle fracture promoted by a decrease in effective stress arising from an increase in fluid pressure (e.g., Sibson et al., 1988; Cox, 1999, 2002; Miller and Nur, 2000).

- Ductile fracture of rock deforming in the viscous-plastic regime (Shigematsu et al., 2001).

- Localisation of rock deforming in the viscous regime due to thermal-viscous coupling between viscous dissipation and strain-rate (Ogawa, 1987; Hobbs and Ord, 1988; Kameyama and Kaneda, 2001; Kameyama, 2002; Tanaka et al., 2002).

To this list, for completeness, although not discussed at the Sendai Conference, should be added unstable sliding in the viscous regime (Bowden-Tabor Friction-see Estrin and Brechet, 1996, for a review). This will be elaborated upon in Section 4 below.

The structure of this discussion is as follows. In Section 2 we consider the background and motivation for Session 7 of the Sendai conference. In particular we concentrate on recent observations in the Sendai region that suggest accelerated motion on a shallowly dipping shear zone (Iio and Kobayashi, 2002) below the base of the seismogenic zone prior to major earthquake activity on a steeply dipping zone in the Upper Crust. Also included are observations by Sacks (see Sacks et al., 1981; Linde et al., 1988) that energy can be stored in the Lower Crust implying that the constitutive behaviour of the Lower Crust is more complicated than that of the Standard Linear Solid (Maxwell linear elastic-viscous behaviour-see Jaeger, 1969). In Section 3 we look at the classical models of the rheological structure of the Earth's crust and see how they might be modified to account for more complicated constitutive relations than just Byerlee and steady state power-law creep behaviour. In Section 4 we discuss unstable sliding in both the plastic and viscous regimes as mechanisms for large earthquakes with emphasis on the recent work by Reinen (2000) and Reinen et al. (1994) and by Estrin and Brechet (1996); this includes ductile failure as an earthquake generating mechanism (Shigematsu et al., 2001). In Section 5 we discuss thermal-viscous coupling as a mechanism for the formation of shear zones in elastic-viscous materials with emphasis on recent work by Cherukuri and Shawki (1995a, b; see also Ogawa, 1987; Hobbs and Ord, 1988; Kameyama and Kaneda, 2001; Tanaka et al., 2002). Finally, in Section 6, we consider how these observations and concepts might be unified into a common framework for future developments in modelling crustal behaviour. 


\section{Background and Motivation}

Iio and Kobayashi (2002), in introducing the Sendai Conference, proposed that seismogenic faults in the upper crust may be associated with localised extensions into the lower, viscous crust and that aseismic accumulation of strain within these aseismic zones ultimately nucleates seismic failure on the upper crustal fault. Furthermore, they proposed that precursor, aseismic slip accelerates prior to the seismic event and that such accelerated motion may be expressed as accelerated tilt and/or distortion at the surface, thereby providing an observable precursor process in advance of a major seismic event.

Perhaps there is an example of such a downward continuation of a seismogenic fault in the Sendai region of NE Japan where the Nagamachi-Rifu Fault can be imaged by seismic methods down to the base of the seismogenic zone. This fault was the site of a magnitude 5 earthquake in 1998 at a depth of $12 \mathrm{~km}$. In addition, seismic studies reveal the existence of a thin, shallow dipping S-wave reflector, below the base of the seismogenic zone, which is interpreted as a fluid filled shear zone (Umino et al., 2002). It is proposed that localised, aseismic shear flow within this shallow dipping zone preceded and nucleated the main seismic event on the Nagamachi-Rifu Fault higher in the crust. Hence, if such mid crustal accelerated creep events could be detected, these events would act as predictors for major upper crustal earthquakes.

Such observations lead to the questions posed in the Introduction to this paper. In particular, we would like to understand the mechanism of shear localisation in the lower, dominantly viscous, crust and how this localisation might lead to a large earthquake event in the upper, dominantly plastic, crust. We also want to understand the angular relationships between faults in the upper crust and shear zones in the lower crust. Associated with these questions is another question regarding the mechanism of earthquake production at the base of the seismogenic zone. Are such events the product of unstable frictional sliding in essentially brittle materials, as is commonly accepted for events in the uppermost crust, or, are other mechanisms possible in this transition region between dominantly plastic and dominantly viscous behaviour (namely, ductile fracture and/or large stress drops associated with localisation in essentially viscous materials)?

These questions raise a number of issues regarding the constitutive behaviour of the crust at various depths. For instance, we shall see that localisation of deformation in a viscous material is not possible unless there is geometrical, chemical or mechanical softening. Hence, if the lower crust is viscous, what is the softening mechanism? Or, perhaps the constitutive behaviour of the lower crust is more complicated than power-law viscous? Perhaps the behaviour of the lower crust is better described as elastic-viscous-plastic? The plastic element could then enable localisation of deformation without the implication of viscous-softening. Even more complicated models exist; examples are given by RegenauerLieb (1999) involving localisation and void formation. The issue is: What mechanisms are responsible for localisation in the lower crust?

Indeed there are observations that do suggest a lower crustal constitutive behaviour more complicated than elastic- viscous. Energy can be stored in the lower crust as was demonstrated in the papers by Sacks et al. (1981) and Linde et al. (1988) who described several examples of slow stable sliding on large faults. The slip regions of these faults are so large that the stress-carrying region must encompass part of the lower crust. An important observation is that the same fault that undergoes stable sliding can behave at another time as an unstable fault where a large earthquake is generated. The implication of these observations is that in the plasticviscous transition zone strain-energy can be stored and ultimately released with both high and low sliding velocities. This indicates a degree of elasto-plasticity (as opposed to pure elastic-viscous behaviour) below the upper crust.

The objective of this paper is to discuss these issues and to arrive at some answers to the questions posed in Section 1 of this paper in an attempt to place the papers presented in Session 7 of the Sendai Conference within an overall framework of coupled thermal-mechanical behaviour.

\section{The Classical Byerlee-Power Law Rheological Structure of the Crust}

Goetze and Evans (1979) proposed that the variation of shear stress with depth in the crust is controlled by brittle behaviour at shallow depths so that the shear stress increases linearly with depth (the Byerlee relationship). This linear variation of shear stress with depth continues down to the depth where the Byerlee relationship intersects the viscous (power-law) behaviour for a prescribed strain-rate for the rock that makes up the lower crust. The shear stress then decreases with increasing depth as viscous behaviour dominates and temperature increases with depth.

This kind of variation of shear stress with depth has been elaborated upon by a large number of authors but the main modification to the original proposition by Goetze and Evans (1979) has been the replacement of a two layer quartzite/peridotite crust by a sandwich structure made up of several layers of different rock types (Bird, 1978; White and Bretan, 1985; Carter and Tsenn, 1987; Ord and Hobbs, 1989). This results in a "Christmas tree" shaped profile of shear stress against depth.

There are a number of comments that need to be made regarding these concepts. These involve:

- Breakdown of the linear Byerlee relationship at high pressures.

- Constitutive relations more general than the power-law viscous relationship.

- The introduction of elasticity into the constitutive relationships.

\subsection{Breakdown of the linear Byerlee relationship}

The extrapolation of the linear Byerlee relationship to great depths such as the Moho or the upper mantle with geothermal gradients of $10-20 \mathrm{C}^{\circ} \mathrm{km}^{-1}$ implies that shear stresses of the order of $1.0 \mathrm{Gpa}$ exist at such depths. Shear stresses of this order of magnitude are unlikely since the intact rock between the pre-existing fractures is likely to fail in a plastic or viscous manner before such high shear stresses are attained. 
The classical Byerlee relationship is in fact a linear MohrCoulomb envelope for the crust with cohesion equal to zero and a friction angle of approximately $40^{\circ}$ independent of depth. Mohr-Coulomb envelopes for most materials become independent of normal stress at high normal stresses (see Paterson, 1978). Thus, it appears unlikely that the linear Byerlee envelope continues to great depths and it is likely that the failure strength ultimately becomes more or less independent of depth.

\subsection{Constitutive relations more general than the power- law relationship for steady state creep}

In the lower parts of the crust the constitutive behaviour is classically represented as a nonlinear viscous power-law relationship during steady state creep. As indicated in Section 5 of this paper, such a material will not localise during deformation unless softening occurs. Localisation in exposed lower crustal materials is ubiquitous (see for instance, Ramsay, 1974) and seismic profiling of the lower crust commonly reveals localisation of deformation (see for instance, Brewer and Smythe, 1984). This means that we need to understand softening mechanisms in viscous materials or address the issue that perhaps lower crustal materials also have a plastic component to their constitutive behaviour so that localisation controlled by yield-surface corner behaviour (see Rudniki and Rice, 1975) or other yield associated behaviour (see Vermeer and de Borst, 1984) can be incorporated into realistic models of the Earth's crust.

There is a very large number of approaches to this problem. For instance, in the paper by Shibazaki et al. (2002) the constitutive behaviour of the crust is modelled as a combination of frictional behaviour (including rate- and statedependant behaviour), exponential creep (at high stresses) and power law creep (at low stresses). In their model, different combinations of these three types of behaviour dominate depending on depth in the crust. Modelling results show details of the relationships between frictional slip in the upper crust and power law dominant ductile flow in the lower crust. The authors demonstrate that this model results in slow but accelerating ductile slip in the lower crust is a precursor to frictional (earthquake) slip in upper crust as proposed by Iio and Kobayashi (2002) in Session 1 of the Sendai Conference. However, such combinations of constitutive behaviour cannot produce localisation unless some kind of softening or yield behaviour is also incorporated.

Clearly a number of other models are possible, the issue is to constrain such models with detailed, but directed, field studies coupled, whereever possible, with seismic imaging of the lower crust.

There are a number of interactions with other processes that need to be investigated. Many of these are prompted by interactions with other sessions at the Sendai Conference, especially the role of fluid pressure cycling and the incorporation of such effects into classical elasto-viscous constitutive laws. Also the analogies drawn by Kameyama in Session 6 of the Sendai Conference between thermal-viscous instabilities and frictional instabilities are particularly useful (see Kameyama, 2002).

Finally the observation that there is a transition zone (which may be quite thick) between the upper elasto-plastic and the lower elasto-viscous crust implies that there are tran- sitional constitutive laws that describe this transition and hence exploring the incorporation of pore pressure and frictional effects into hybrid elasto-viscous-plastic constitutive laws is worth some effort. As indicated, there were several treatments of this in Session 7 but the remaining link to constitutive laws which involve dilatant flow of elastic-viscousplastic materials with a consequent dependence upon effective stress through fluid pressure was not made, but needs to be made, explicitly. An attempt along these lines is the paper by Regenauer-Lieb (1999), where localisation in the lower crust was explored in association with the transport of $\mathrm{CO}_{2}$ through the crust.

\subsection{Elasticity in the constitutive relationships}

To date, there has been relatively little attempt to incorporate constitutive relations for crustal materials more complicated than the linear Byerlee relationship and the nonlinear, viscous power-law relationship. Neither of these explicitly involves elasticity. The oft-quoted rationale is that elasticity does not need to be incorporated since the natural deformation process is slow and therefore intrinsically controlled by plastic or viscous behaviour; the elastic deformation is small compared to the plastic or viscous part of the total deformation. This rationale is true so long as the deformation remains homogenous with no development of instabilities such as fractures, folds or shear zones - structures that are ubiquitous in crustal rocks at all depths.

A useful concept here is that of the Deborah Number, $D_{e}$, defined as the ratio of the time scale for the process of interest to the stress relaxation time scale. The common geological argument that elasticity can be neglected is an assertion that the Deborah Number is much less than one. This is equivalent to saying that the stress relaxation time scales are always large in comparison to the time scales for the growth of instabilities in the deformation with the resulting corollary that geological deformations must always be homogeneous; this quite clearly is not true. Instabilities such as folds and shear zones grow and evolve at their own separate time scales and the mere fact that they are ubiquitous in deformed rocks means that the time scales for the growth of these instabilities exceed the stress relaxation time scales for the material; that is, $D_{e}$ is greater than one for many geological processes. Neglect of instantaneous aspects of material behaviour such as elasticity and most types of plasticity, where the constitutive behaviour is rate insensitive, is admissible only if $D_{e} \ll 1$.

An important part of modelling realistic behaviour of the crust is to incorporate elasticity into crustal models together with plasticity so that generalised elastic-viscousplastic constitutive relations are used. Some advances along these lines for crustal and mantle materials have been made by Moresi and Solomatov (1998), Regenauer-Lieb and Yuen (2000a), Branlund et al. (2000), Hobbs et al. (2000), and Moresi et al. (2001).

\section{Unstable Sliding in both the Plastic and Viscous Regimes}

Early experiments on frictional sliding in the brittle regime have established that the coefficient of friction depends on two parameters: the velocity of sliding and the physical state of the sliding surface (see Dieterich, 1979, 1981; Ruina, 1983). It has become clear from this and subsequent work 
that unstable sliding in the brittle regime arises when there is velocity weakening of the coefficient of friction (see $\mathrm{Gu}$ et al., 1984). The issue seems to be to gain a clear understanding of the conditions under which surface state dependent frictional behaviour (as opposed to a dependence solely upon the sliding velocity) is dominant together with those conditions under which (unstable) velocity weakening state behaviour as opposed to (stable) velocity strengthening state behaviour develops.

At the Sendai Conference, Reinen elaborated upon her multi-mechanism frictional constitutive model (see also Reinen et al., 1994; Reinen, 2000) where, at low velocities, the material exhibits only rate-strengthening behaviour with no dependence on surface state variables. Since the coefficient of friction in this regime depends only on the velocity of sliding this behaviour is viscous (termed here the flow regime). At higher velocities, the sliding behaviour of the material is the classical Dieterich-Ruina behaviour where the coefficient of friction depends not only on the velocity of sliding but also on the state of the sliding surface. This is termed here the state variable regime. In this regime the velocity dependence of the coefficient of friction can be strengthening or weakening, again for reasons that are not clear at present.

Reinan shows that sometimes, in numerical modelling experiments, it is possible to continue along what seems to be a velocity-strengthening branch (the flow regime) as the velocity of sliding increases past where one would expect the state variable regime to begin; this situation eventually collapses onto the state variable branch. In other examples, there is a more or less smooth transition from the flow regime branch at low velocities to the state variable branch at higher velocities. Modelling results by Reinen simulate slow, stable fault slip, high speed (earthquake) slip and combinations of the two with stable slip preceding large stress drops associated with high speed slip. Perhaps the observation of Sacks et al. (1981) that some faults slide in a stable manner at one time but in an unstable manner at other times is a manifestation of this behaviour.

The spring-slider system, as modelled by Reinan, seems to show that the transition from the flow regime to the state variable regime is a bifurcation with the precise behaviour at and after the bifurcation point governed by the stiffness characteristics of the system and perhaps by the sliding velocity. This type of bifurcation behaviour has not been formally investigated (in a mathematical sense) for this system.

The type of behaviour described by Reinan, where there is a transition from purely viscous behaviour at low sliding velocities to viscous behaviour with an associated dependence on the state of the sliding surface, is identical to the behaviour discussed, in a wider context, by Estrin and Brechet (1996). In the case of brittle material (Byerlee Material, in the terminology of Estrin and Brechet) it is proposed that at low sliding velocities the dominant mechanism operating is the tensile fracture of welded asperities at the sliding surface. Here the velocity dependence of the tensile strength is the only important process and since the tensile strength of most materials increases with strain-rate (see for example Ashby and Jones, 1986), the coefficient of friction increases with velocity. At higher rates of sliding where the coefficient of friction depends on both the sliding velocity and the state of the surface, asperities are smoothed out during sliding, the area of asperity surface and the degree of welding being larger for slower sliding rates. Here, the coefficient of friction is larger for larger asperities and for more strongly welded surfaces so that a velocity weakening effect results. This velocity weakening effect is applicable to brittle materials and is finally offset at faster sliding rates by the simple crushing of asperities where again the strain rate dependence of the tensile fracture strength dominates; asperities have no time to distort and/or weld and the coefficient of friction therefore again increases with increasing velocity.

Estrin and Brechet (1996) point out that, in principle, the same velocity dependent characteristics applicable to brittle materials apply to sliding of surfaces in situations where crystal plasticity dominates the deformation processes (Bowden-Tabor Material). In this case, for low and high sliding velocities, the velocity dependence arises solely from the strain rate dependence of the local shear stress and hence increases with increasing sliding velocity. For intermediate sliding rates, local flattening of asperities plays an important role with larger flattening for slower sliding rates, resulting in a decrease in the coefficient of friction with increasing sliding rates. These observations are important in understanding how unstable sliding can arise in situations where only crystal plastic deformation mechanisms are in operation). The proposal that velocity weakening can occur in materials under conditions where otherwise "ductile" behaviour dominates is an important mechanism to consider for earthquake generation at mid-crustal depths. Such considerations may be relevant to the observations presented during Session 7 of the Sendai Conference, where evidence was given of failure of mylonites by ductile fracture (Shigematsu et al., 2001).

\section{Instabilities Arising from Thermal-Viscous Coupling}

This section is concerned with three questions. Firstly, what are the processes responsible for the localisation of deformation in the essentially viscous part of the crust? Secondly, are there mechanisms for earthquake generation in this part of the crust? Thirdly, if localisation of deformation occurs in the essentially viscous part of the crust, what controls the orientation of the resulting shear zone and is it parallel or can it be highly inclined to an associated highly dipping fault or shear zone in the upper crust?

As indicated in Section 3, the classical view of the lower crust is that of a viscous power-law material. Such materials do not localise during deformation unless strain softening occurs (see Anand et al., 1987). Since downward continuation, into the lower crust, of large faults is documented at many places on Earth (for example, the North Sea, Reston, 1990; San Andreas in some places, Zhu, 2000; Central Australia, Goleby et al., 1989), two conclusions are possible. Either the lower crust does behave as a simple viscous, power-law material but strain-softening is important, caused by chemical, microstructural or thermal weakening, or deformation induced damage or, the lower crust does not behave as a simple viscous, power-law material and some form of yield (i.e. plasticity) is involved. 


\subsection{Softening of an elastic-viscous material}

Although chemical and microstructural softening of viscous materials due to changes of grain size, chemical composition and/or mineralogy within shear zones is undoubtedly an important process (see for instance, Rutter, 1999; Regenauer-Lieb et al., 2001), the Sendai Conference concentrated on softening resulting from viscous dissipation and from deformation induced damage, in particular, void formation leading to ductile fracture. The gently dipping feature imaged below the Nagamachi-Rifu Fault in the Sendai region is commonly interpreted as a fluid filled shear zone but this still poses the question of the mechanism of localisation and of concentrating fluids within the zone of localisation. Below we concentrate more on processes of localisation controlled by thermal softening.

5.1.1 Thermal softening Localisation of deformation in rate insensitive, plastic materials has had a long history of study (see the detailed review by Shawki and Clifton, 1989). Localisation occurs in these materials once the mathematical conditions for bifurcation are satisfied. This occurs when there is a loss in ellipticity in the governing rate equations describing the deformation. Physically this commonly happens when the strain-hardening modulus attains a critical value (not necessarily negative - see Rudniki and Rice, 1975). At that stage an initially homogeneously deforming material can continue to deform homogeneously or the deformation can continue on another path (i.e. the behaviour bifurcates) and the material deforms inhomogenously through the localisation of deformation. In rate insensitive, plastic materials both the time and the location of the localisation are defined precisely by the time and location of the mathematical loss of ellipticity of the underlying governing equations. Another discussion of localisation in plastic materials with an emphasis on the yield surface is given by Hobbs et al. (1990).

In rate sensitive materials the systematics of the localisation process has not been as well understood until relatively recently (see Cherukuri and Shawki, 1995a, b). The critical aspect of bifurcation behaviour in rate sensitive materials is that the formation of instabilities is associated with dissipative mechanisms such as heat diffusion and viscosity. Once these mechanisms are introduced, the occurrence of localisation is not so clearly defined as in rate insensitive, plastic materials. Such dissipative mechanisms delay the occurrence of severe localisation, after the necessary mathematical conditions for localisation have been met, and tend to smooth the process out. Cherukuri and Shawki (1995a) show that the history of the total kinetic energy of the deforming body is sufficient not only to describe the conditions for initiation of localisation in power-law viscous materials undergoing simple shearing deformations but to describe the subsequent development of the localisation as well.

The localisation of deformation in rate sensitive materials depends upon feedback between the heat generated by viscous dissipation and the temperature dependence of viscosity. If the deformation rate is fast enough, locally, lack of time for heat conduction results in the temperature being highest in regions of highest strain-rate. In rate sensitive materials, the strong dependence of viscosity upon temperature leads to locally intense deformation where the temperature is highest, thus increasing the temperature further. The thermal softening process is counterbalanced by strain- and strain-rate hardening. Thus a global softening, and hence, localisation, depends upon the thermal softening exceeding the mechanical hardening (see Poirier et al., 1979; Poirier, 1980; Hobbs et al., 1986; Ogawa, 1987; Hobbs and Ord, 1988).

Cherukuri and Shawki (1995a, b) show that the history of localisation in purely viscous (no elasticity) materials can be characterised by three dimensionless numbers:

- the diffusion number, $r_{0}$, which is the ratio of measures of viscous and thermal diffusion effects,

- the inertia number, $\rho_{0}$, which is the ratio of inertial to viscous effects, and,

- the dissipation number, $r_{1}$, which is the ratio of the thermal energy density produced during some reference time, to the energy required to raise the temperature of a unit volume through some reference temperature.

In addition, Kameyama and Kaneda (2001) have analysed the conditions for instability in an elastic-viscous material, neglecting the effects of inertia. They identify two dimensionless numbers (formerly discussed by Ogawa, 1987) that characterise the localisation of such materials. These are:

- A number, $g$, which is the ratio of the rate of temperature rise due to viscous dissipation to the rate of conductive cooling; this is similar to the diffusion number of Cherukuri and Shawki discussed above.

- A number, $f$, which is the ratio of the elastic stiffness to the viscous stiffness; such a term does not enter into the discussions of Cherukuri and Shawki (1995a, b) since they did not consider the influence of elasticity.

A zero or negative strain hardening modulus is a necessary condition for instability (as pointed out in the paper by Kameyama and Kaneda (2001) in Session 6 of the Sendai Conference) but it is not sufficient. As indicated above, the inclusion of dissipative processes means there is commonly a time lag between the mathematical conditions for instability being met and the instability starting to propagate. Cherukuri and Shawki (1995a, b) distinguish two critical times that describe the history of localisation in thermal softening materials. One is the critical time, $t_{c r}$, that elapses between some perturbation in the deformation and the onset of localisation. This onset of localisation corresponds to a maximum in the time derivative of the total kinetic energy. The second is the stabilisation time, $t_{\mathrm{stb}}$, which measures the time when the localisation stops growing. This is when enough time has elapsed that the conduction of heat away from the zone of localisation has become sufficient to inhibit thermal softening and corresponds to the time derivative of the total kinetic energy decreasing back to zero. The dependences of $t_{c r}$ and of $t_{\text {stb }}$ upon $r_{0}$, are shown in Fig. 1.

Cherukuri and Shawki also discuss the increase in thickness of a zone of localisation between the time of formation, and the time the shear zone stops growing. The results are similar to those of Tanaka et al. (2002) who examine the growth in thickness of a shear zone in a viscous material due to moment diffusion during isothermal shearing deformation. The growth in thickness behaves in a manner simi- 


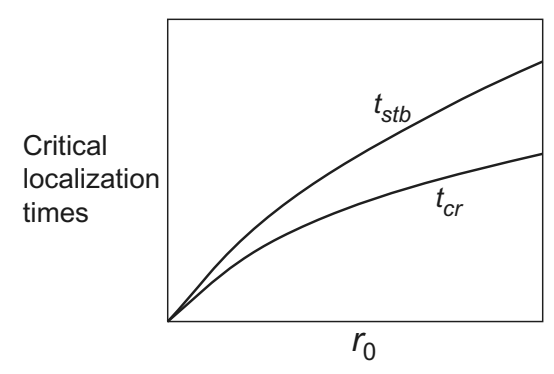

Fig. 1. Dependence of $t_{c r}$ and $t_{\text {stb }}$ upon the diffusion number, $r_{0}$. After Cherukuri and Shawki (1995b).

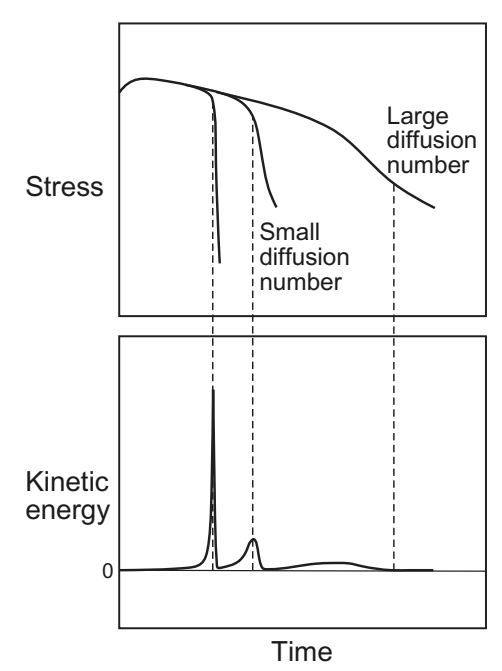

Fig. 2. Generalised dependence of the evolution of stress (and hence of $t_{c r}$ ) upon the diffusion number. After Cherukuri and Shawki (1995b).

lar to heat conduction in that the thickness increases with the square root of the time. This relationship is applied to a natural shear zone associated with the Hatagawa Fault Zone to give a time of formation of $760 \mathrm{~s}$ for the $0.076 \mathrm{~m}$ thick shear zone. The associated creep velocity is comparable with that observed during interseismic periods.

Figure 2 shows the evolution of stress, and hence the dependence of $t_{c r}$, following a perturbation in the system, upon the diffusion number. For small diffusion numbers in a system where the stress is softening with time, localisation follows rapidly after the perturbation and the stress drop is catastrophic. This corresponds to a seismic event in essentially viscous material. For progressively larger diffusion numbers, the lag between perturbation and localisation is longer and the ultimate stress drop is less catastrophic corresponding to aseismic shearing. Finally, for large enough diffusion numbers, there may be no localisation on very large time scales.

Figures 3 and 4 show the results of some calculations for simple shearing of peridotite at a number of temperatures (corresponding to different diffusion numbers). In these examples, for peridotite at $950^{\circ} \mathrm{C}$ and $10^{-14} \mathrm{~s}^{-1}$ strain-rate, this time lag between a perturbation in strain rate and catastrophic stress drop is of the order of $10^{5}$ years. Thus, in this case, if a perturbation in strain rate arises from a slight change in plate motion, there may be a lag of $10^{5}$ years before seismic activity ensues. A similar lag in the propagation of a localised zone of deformation arising from the feedback relation between viscous dissipation and viscosity is discussed by Regenauer-Lieb and Yuen (2000b).

5.1.2 Orientation of zones of localisation An important issue for the Sendai Conference was to understand the control on the orientation of zones of localisation particularly in the mid to lower crust. The following is a discussion of these controls.

For most materials there is a fairly direct relationship between the orientation of a shear zone and the local orientation of the maximum compressive stress responsible for deformation (see Anand et al., 1987).

Consider a viscoplastic material (no elasticity), for which the shear stress, $\tau$ is given by $\tau=\tau(\gamma, \dot{\gamma}, T, p)$. where $\tau$ is the shear stress, $\gamma$ is the shear strain, $\dot{\gamma}$ is the shear strain rate, $T$ is the temperature and $p$ is the mean stress (the stress deviator). If we write the pressure hardening as $P=\partial \tau / \partial p$, then the angle, $\theta$ between the shear zone normal and the maximum principal stress is given by

$$
\theta= \pm\{(\pi / 4)+(\beta / 2)\}
$$

where

$$
\beta=\sin ^{-1}\left[\left(1 / P^{o}\right)\left\{1-\left(1-P^{o 2}\right)^{1 / 2}\right\}\right]
$$

Here, $P^{o}$ is the pressure hardening, evaluated whilst the material is undergoing homogeneous deformation.

The expression for $\theta$, above, translates, for the classical Mohr Coulomb material, to:

$$
\theta= \pm\{(\pi / 4)+(\phi+\psi) / 4\}
$$

where $\phi$ is the friction angle and $\psi$ is the dilation angle (Vermeer and de Borst, 1984).

Since $\beta$ is rarely larger than perhaps $20^{\circ}, \theta$ tends to be in the range $55^{\circ}$ to $45^{\circ}$. This means that if the maximum compressive stress has the same orientation in the upper and lower crust then zones of localised deformation must be approximately parallel to each other. An exception would occur if the crust was strongly layered in a rheological sense. Then, if a very strong layer underlies a very weak layer undergoing localisation then the boundary between these two layers may "detach" over a limited distance and behave as a shear zone with the local maximum principal stress at $45^{\circ}$ to the resulting shear zone. In such an example a steeply dipping shear zone in the upper crust may pass downward in a listric manner into the "detachment" zone.

In Fig. 5 we show the results of modelling the deformation of an initially $30 \mathrm{~km}$ thick crust composed of an upper crust, initially $15 \mathrm{~km}$ thick and comprised of Mohr Coulomb material, underlain by a lower crust with elastic-power-law viscous material. The model is shortened horizontally with thermal-viscous coupling included. Zones of localised strain develop in the upper crust that extend down to the base of the Mohr Coulomb material; the deformation is broadly distributed in the lower crust and there is no downward extension of the upper crustal shear zones. The downward continuation of steep faults into the lower crust as low dipping zones of localised flow (as indicated for instance in the paper 

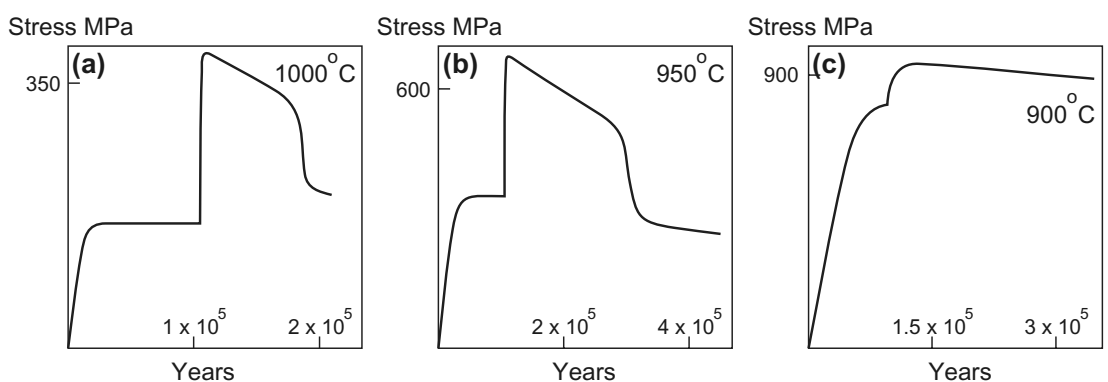

Fig. 3. Evolution of shear stress for a peridotite undergoing a simple shearing deformation history. In each example the ambient temperature is (a) $1000^{\circ} \mathrm{C}$, (b) $950^{\circ} \mathrm{C}$, (c) $900^{\circ} \mathrm{C}$. The initial steady state strain rate is $10^{-14} \mathrm{~s}^{-1}$ but this is instantaneously perturbed to $5 \times 10^{-14} \mathrm{~s}^{-1}$ resulting in a stress increase which then decays with time due to viscous dissipation until (with time) the stress evolves according to the format outlined in Fig. 2. Constitutive parameters are those of the "wet" Aheim Dunite of Chopra and Paterson (1981). Calculations for this figure and for Figs. 4 and 5 performed using the finite difference code FLAC (Cundall and Board, 1988).
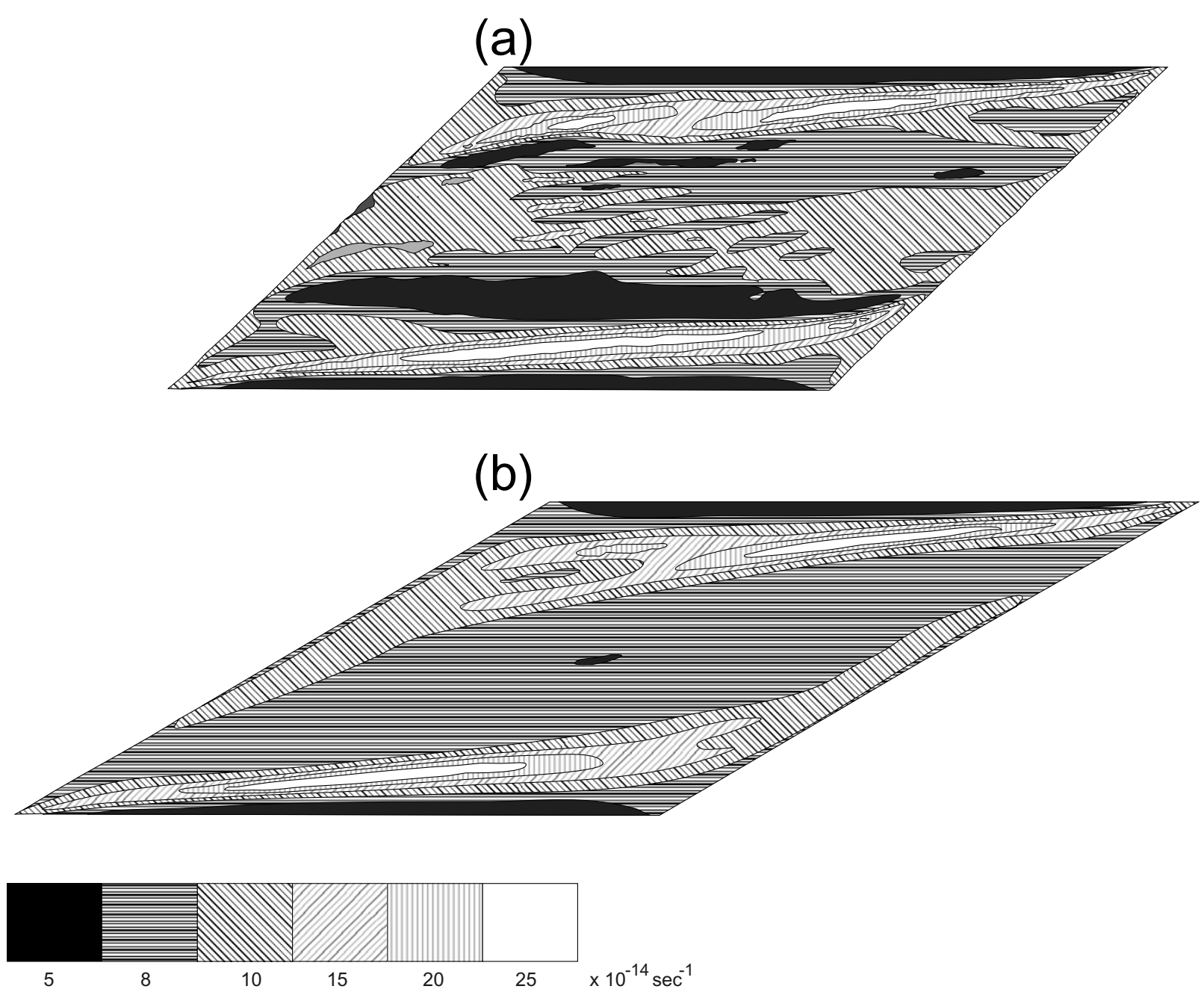

Fig. 4. Simple shearing of a peridotite (constitutive parameters are those of "wet" Aheim Dunite of Chopra and Paterson (1981). Initial strain rate is $10^{-14} \mathrm{~s}^{-1}$ at $950^{\circ} \mathrm{C}$ ambient temperature. The strain rate is perturbed to $5 \times 10^{-14} \mathrm{~s}^{-1}$ at the ambient temperature of $950^{\circ} \mathrm{C}$. Viscous dissipation results in localisation of deformation resulting in local strain rates of $2.5 \times 10^{-13} \mathrm{~s}^{-1}$ and local temperatures in both (a) and (b) of $1060^{\circ} \mathrm{C}$ within the shear zones. In (a) the time after perturbation of the strain rate is 200,000 years whilst in (b) this time is 400,000 years.

by Iio and Kobayashi (2002) in Session 1 and in the posters) will only occur if the lower crust is undergoing horizontal simple shearing whilst the upper crust is undergoing a horizontal shortening. This could be the situation in the Sendai region above a subduction zone. As indicated above how- ever, another explanation may involve rapid downward variations in strength within the crust.

\subsection{Ductile fracture}

The paper by Shigematsu et al. (2001) proposes a mechanism of failure in the plastic-viscous transition zone due to 
(a)
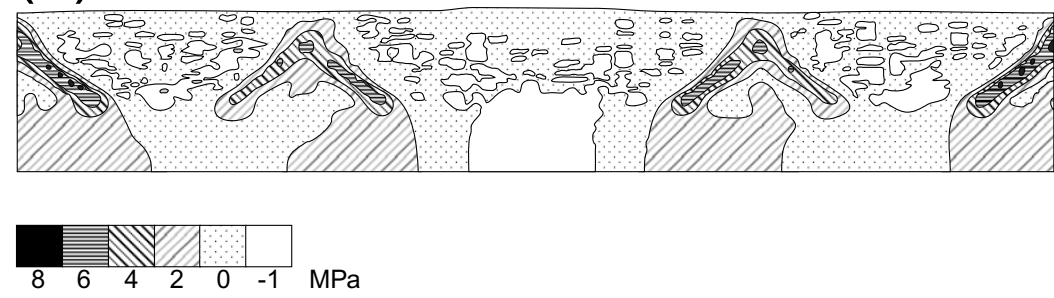

(b)
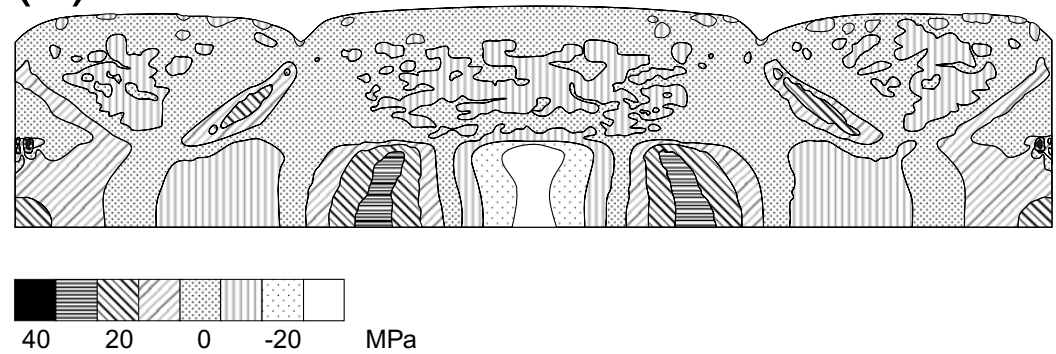

Fig. 5. Deformation of a crustal section initially $200 \mathrm{~km}$ long and $30 \mathrm{~km}$ thick. Temperature at the base of the crust is held constant at $900^{\circ} \mathrm{C}$ and that at the top, $0^{\circ} \mathrm{C}$. The top initial $15 \mathrm{~km}$ is comprised of Mohr-Coulomb material with cohesion of $100 \mathrm{Mpa}$ and friction angle of $30^{\circ}$, independent of depth. The lower crust is comprised of elastic-power-law viscous material with constitutive parameters corresponding to those of the "dry" anorthosite of Shelton and Tullis (1981). The plots show contours of $\sigma_{y y}$ where the $y$-axis is vertical. Localisation occurs in the upper crust but transfers to distributed deformation in the lower crust. In (a) the shortening is $1.6 \%$ whilst in (b) the shortening is $8 \%$. The strain rate is $2 \times 10^{-14} \mathrm{~s}^{-1}$ in (a) and $4 \times 10^{-14} \mathrm{~s}^{-1}$ in (b).

ductile fracture. Electron microscope observations of microstructures in mylonites of the Hatagawa Fault Zone reveal fracturing that nucleates in regions of high microvoid concentrations. It is proposed that these microvoids form in response to strain incompatibilities that develop at grain boundaries due to dislocation pile-up. Such structures are preferentially developed along C-planes in these S-C mylonites.

The proposal that earthquakes can be generated by unstable sliding on fractures initiated during ductile fracture implies unstable sliding in the viscous or plastic regimes and hence a connection to the Bowden Tabor frictional processes outlined in Section 4. This unstable sliding process assumes that asperities exist under such mid-crustal conditions. This, in turn, implies the existence of spaces between the asperities, which presumably are filled with overpressured fluid in order to keep the spaces between asperities open. This coupling between fluids and viscous deformation is a field which has not received much attention in the literature to date and the relevant constitutive relations need to be explored. An example is the use of the Gurson and Tvergaard constitutive relations by Regenauer-Lieb (1999).

\section{A Framework for Future Developments}

In addressing the questions posed in the introduction to this paper it becomes clear that one has to adopt a more general view of the constitutive properties of both the upper and lower crust than the classical view consisting of the Byerlee Relationship for the upper crust and power law viscous behaviour for the lower crust. Additional constitutive properties include:
- Unstable frictional sliding in both the brittle (Byerlee Friction) regime and the viscous-plastic (BowdenTabor) regime. In both of these regimes, but particularly for the latter regime, the role of fluids in the crust in supporting an otherwise open network between asperities becomes important. In the upper crust, coupling between fluid pressures and mechanical behaviour is well known (e.g., Sibson et all., 1988; Cox, 1999; Miller and Nur, 2000), but for lower crustal materials in which the intrinsic porosity is small, there has been relatively little attention given. An exception is the paper by Regenauer-Lieb (1999) in his study of carbon dioxide transport through lower crustal rocks in the Rhine Grabben. He adopted a constitutive relationship for lower crustal rocks in which porosity generation occurs concurrently with viscous-plastic deformation. Clearly there is a wealth of work to be done here to understand the coupling between dilation, fluids and the constitutive relations for lower crustal rocks and the controls on localisation and unstable sliding.

- Feedback between dissipative heating and viscous strength and the role this process plays in localisation of deformation.

- Consideration of more complicated constitutive relations that represent the transition from elasto-plastic upper crust to elasto-viscous lower crust.

The conclusions that arise from this discussion are:

1. Assuming that the lower crust is essentially elastoviscous in its constitutive relation, deformation is localised in the lower crust under conditions where soft- 
ening occurs. Such softening can arise from chemical processes, such as water-weakening, microstructural evolution, due to grain-size reduction, damage accumulation, such as may be associated with void formation and micro-cracking, and thermal softening due to viscous dissipation. A classical power-law viscous material will not localise unless such softening behaviour occurs. Deformation in a classical power-law material, without such softening is always distributed. It is possible, however, that the constitutive behaviour is more complicated than just elasto-viscous and that some plasticity (or yield) behaviour is present. In this case localisation could be ubiquitous in such materials once yield was attained.

2. If the history of deformation is similar in both the upper and lower crust (that is, the orientations of stress are similar) then the localised downward extensions of seismogenic faults into the lower crust have a geometry that is similar to that of seismogenic faults in the upper crust. The orientation of the stress field controls the orientation of the planes of localisation. One explanation for there to be a large angle between zones of localisation in the upper and lower crusts there must be a strong difference in the orientation of the stress field in the upper and lower crust. This would be the case for instance if the upper crust is in horizontal compression whilst the lower crust undergoes a horizontal simple shearing. Such could be the situation above a subduction zone as at Sendai. Another explanation relies upon strong contrasts in strength within the crust so that "detachment" occurs upon the boundary between two contrasting strength regimes.

3. Several mechanisms were identified as potentially involved in the faulting process associated with large earthquakes near the base of the seismogenic zone. These mechanisms include Byerlee Friction, with associated state and velocity dependent weakening, and instabilities arising from thermal softening and ductile failure.

4. All of these processes are capable of producing accelerated aseismic slip on the downward extensions of faults before large intraplate earthquakes occur. More detailed modelling (with high spatial resolution) of individual geometries is essential before one can be definitive about whether accelerated slip in mid crustal regions constitutes an important, measurable precursor to large earthquakes.

Acknowledgments. I would like to thank Professor Shimamoto and an anonymous reviewer for helpful comments upon this paper. I would also like to thank the organisers of the Sendai Conference for the opportunity to be within $15 \mathrm{kms}$ of the base of the seismogenic zone in Japan.

\section{References}

Anand, L., K. H. Kim, and T. G. Shawki, Onset of shear localization in viscoplastic solids, J. Mech. Phys. Solids, 35(4), 407-429, 1987.

Ashby, M. F. and D. R. H. Jones, Engineering Materials 2, Pergamon Press, Oxford, 1986
Backofen, W. A., Deformation Processing, 326 pp., Addison-Wesley, 1972. Bird, P., Initiation of intracontinental subduction in the Himalaya, J. Geophys. Res., 83, 4975-4987, 1978.

Branlund, J., K. Regenauer-Lieb, and D. A. Yuen, Fast ductile failure of passive margins for sediment loading, Geophys. Res. Lett., 25(13), 19891992, 2000.

Brewer, J. A. and D. K. Smythe, MOIST and the continuity of crustal reflector geometry along the Caledonian-Appalachian orogen, J. Geol. Soc. London, 141, 105-120, 1984.

Byerlee, J. D., Theory of friction based on brittle fracture, J. Appl. Phys., 38, 2928-2934, 1967.

Carter, N. L. and M. C. Tsenn, Flow properties of continental lithosphere, Tectonophys., 136, 27-63, 1987.

Cherukuri, H. P. and T. G. Shawki, An energy-based localization theory: 1 Basic Framework, Int. Journ. Plasticity, 11(1), 15-40, 1995a.

Cherukuri, H. P. and T. G. Shawki, An energy-based localization theory: II. Effects of diffusion, inertia and dissipation numbers, Int. Journ. Plasticity, 11(1), 41-64, 1995b.

Chopra, P. N. and M. S. Paterson, The experimental deformation of dunite, Tectonophys., 78, 453-473, 1981.

Cox, S. F., Deformational controls on the dynamics of fluid flow in mesothermal gold systems, in Fractures, Fluid Flow and Mineralization, edited by K. McCaffrey, L. Lonergan, and J. Wilkinson, Geological Society, London, Special Publications, 155, 123-140, 1999.

Cox, S. F., Fluid flow in mid- to deep crustal shear systems: Experimental constraints, observations on exhumed high fluid flux shear systems, and implications for seismogenic processes, Earth Planets Space, 54, this issue, $1121-1125,2002$

Cundall, P. A. and M. Board, A microcomputer program for modelling large-strain plasticity problems, Proc. 6th International conf. On Numerical Methods in Geomechanics, Balkema, Rotterdam, 2101-2108, 1988.

Dieterich, J. H., Modelling of rock friction: 1. Experimental results and constitutive equations, J. Geophys. Res., 84, 2161-2168, 1979.

Dieterich, J. H., Constitutive properties of faults with simulated gouge, in Mechanical Behaviour of Crustal Rocks: The Handin Volume, Geophys. Monogr. 24, edited by N. L. Carter et al., pp. 103-120, AGU, Washington D.C., 1981.

Estrin, Y. and Y. Brechet, On a model of frictional sliding, $P A G E O P H, 147$, 4, 1996.

Goetze, C. and B. Evans, Stress and temperature in the bending lithosphere as constrained by experimental rock mechanics, Geophys. J. R. Astron. Soc., 59, 463-478, 1979.

Goleby, B. R., R. D. Shaw, C. Wright, B. L. N. Kennett, and K. Lambeck, Geophysical evidence of 'thick-skinned' crustal deformation in central Australia, Nature, 337, 325-330, 1989.

Gu, J.-C., J. R. Rice, A. I. Ruina, and S. T. Tse, Slip motion and stability of a single degree of freedom elastic system with rate and state dependent friction, J. Mech. Phys. Solids, 32, 167-196, 1984.

Hobbs, B. E. and A. Ord, Plastic instabilities - implications for the origin of intermediate and deep focus earthquakes, J. Geophys. Res., 93, 1052110540,1988

Hobbs, B. E., A. Ord, and C. Teyssier, Earthquakes in the ductile regime?, $P A G E O P H, \mathbf{1 2 4}, 1 / 2,1986$.

Hobbs, B. E., H.-B. Muhlhaus, and A. Ord, Instability, softening and localization of deformation, in Deformation Mechanisms, Rheology and Tectonics, edited by R. J. Knipe and E. H. Rutter, Geol. Soc. Spec. Pub., 54, 143-165, 1990 .

Hobbs, B. E., H.-B. Muhlhaus, A. Ord, and L. N. Moresi, The influence of chemical migration upon fold evolution in multi-layered materials, in Selbstorganisation. Band II. Non-equilibrium processes and dissipative structures in geoscience, edited by H.-J. Krug and J. H. Kruhl, Duncker \& Humblot, Berlin, pp. 229-252, 2000.

Iio, Y. and Y. Kobayashi, A physical understanding of large intraplate earthquakes, Earth Planets Space, 54, this issue, 1001-1004, 2002.

Jaeger, J. C., Elasticity, Fracture and Flow, Methuen \& Co., London, 268 pp., 1969.

Kameyama, M., On the relevance of thermal viscous coupling as a model of frictional constitute relationship, Tectonophys., February, 2002 (submitted).

Kameyama, M. and Y. Kaneda, Thermal-mechanical coupling in shear deformation of viscoelastic material as a model of frictional constitutive relations, Pure Appl. Geophys., 2001 (in press).

Linde, A. T., K. Suyehiro, S. Miura, I. S. Sacks, and A. Takagi, Episodic aseismic earthquake precursors, Nature, 334, 513-515, 1988. 
Miller, S. and A. Nur, Permeability as a toggle switch in fluid-controlled crustal processes, Earth Plan. Sci. Lett., 183, 133-146, 2000.

Moresi, L. and V. Solomatov, Mantle convection with a brittle lithosphere: thoughts on the global tectonic styles of the Earth and Venus, Geophys. J. Int., 133, 669-682, 1998.

Moresi, L., F. Dufour, and H. Muhlhaus, Mantle convection models with viscoelastic/brittle lithosphere: Numerical methodology and plate tectonic modeling, PAGEOPH, 2001 (in press).

Ogawa, M., Shear instability in a viscoelastic material as the cause of deep focus earthquakes, J. Geophys. Res., 92, 13801-13810, 1987.

Ord, A. and B. E. Hobbs, The strength of the continental crust, detachment zones and the development zones and the development of plastic instabilities, Tectonophys., 158, 269-289, 1989.

Paterson, M. S., Experimental Rock Deformation-The Brittle Field, edited by W. von Engelhardt, Tubingen - T. Hahn, Aachen, Springer-Verlag, 1978.

Poirier, J. P., Shear localization and shear instability in materials in the ductile field, J. Struct. Geol., 2, 135-142, 1980

Poirier, J., J. L. Bouchez, and J. J. Jonas, A dynamic model for aseismic ductile shear zones, Earth Planet. Sci. Lett., 43, 441-453, 1979.

Ramsay, J. G., Folding and fracturing of rocks, 568 pp., Ed. Frank Press, MacGraw-Hill, 1974.

Regenauer-Lieb, K., Dilatant plasticity applied to Alpine Collision: ductile void growth in the intraplate area beneath the Eifel Volcanic Field, J. Geodynam., 27, 1-21, 1999.

Regenauer-Lieb, K. and D. A. Yuen, Fast mechanisms for the formation of new plate boundaries, Tectonophys., 322, 53-67, 2000a.

Regenauer-Lieb, K. and D. Yuen, Quasi-adiabatic instabilities associated with necking processes of an elasto-viscoplastic lithosphere, Physics of the Earth and Planetary Interiors, 118, 89-102, $2000 \mathrm{~b}$.

Regenauer-Lieb, K., D. A. Yuen, and J. Branlund, The initiation of Subduction: Criticality by addition of water, Science, 294, 578-580, 2001.

Reinen, L. A., Slip styles in a spring-slider model with a laboratory-derived constitute law for serpentinite, Geophys. Res. Lett., 27(14), 2037-2040, 2000.

Reinen, L. A., J. D. Weeks, and T. E. Tullis, The frictional behaviour of lizardite and antigorite serpentinites; experiments, constitutive models, and implications for natural faults, Pure Appl. Geophys., 143, 317-358, 1994.

Reston, T. J., Mantle shear zones and the evolution of the northern North Sea basin, Geology, 18, 272-275, 1990.

Rice, J. A., N. Lapusta, and K. Ranjith, Rate and state dependent friction and the stability of sliding between elastically deformable solids, J. Mech. Phys. Solids, 49, 1865-1898, 2001.

Rudniki, J. W. and J. R. Rice, Conditions for the localization of deformation in pressure-sensitive dilatant materials, J. Mech. Phys. Solids, 23, 371-
394, 1975.

Ruina, A. L., Slip instability and state variable friction laws, J. Geophys. Res., 88, 10359-10370, 1983.

Rutter, E. H., On the relationship between the formation of shear zones and the form of the flow law for rocks undergoing dynamic recrystallization, Tectonophys., 303, 147-158, 1999.

Sacks, I. S., A. T. Linde, J. A. Snoke, and S. Suyehiro, A slow earthquake sequence following the Izu-Oshima Earthquake of 1978, in Earthquake Prediction: An International Review, edited by D. W. Simpson and P. G. Richards, Maurice Ewing Series, 4, AGU, 617-628, 1981.

Shawki, T. G. and R. J. Clifton, Shear band formation in thermal viscoplastic materials, Mech. of Materials, 8, 13-43, 1989.

Shelton, G. and J. Tullis, Experimental flow laws for crustal rocks, EOS Trans. Am. Geophys. Union, 62, 396, 1981.

Shibazaki, B., H. Tanaka, H. Horikawa, and Y. Iio, Modeling slip processes at the deeper part of the seismogenic zone using a constitutive law combining friction and flow laws, Earth Planets Space, 54, this issue, 12111218, 2002.

Shigematsu, N., K. Fujimoto, and T. Ohtani, Plastic deformation and fracturing: a case study in the Hatagawa Fault Zone, Proceedings of the International Symposium on Slip and Flow Processes in and below the Seismogenic Region, Sendai, Japan, 2001.

Sibson, R. H., F. Robert, and K. H. Poulson, High-angle reverse faults, fluidpressure cycling, and mesothermal gold deposits, Geology, 16, 551-555, 1988.

Tanaka, H., B. Shibazaki, N. Shigematsu, K. Fujimoto, T. Ohtani, Y. Miyashita, T. Tomita, K. Omura, Y. Kobayashi, and J. Kameda, Growth of plastic shear zone and its duration inferred from theoretical consideration and observation of an ancient shear zone in the granitic crust, Earth Planets Space, 54, this issue, 1207-1210, 2002.

Umino, N., H. Ujikawa, S. Hori, and A. Hasegawa, Distinct S-wave reflectors (bright spots) detected beneath the Nagamachi-Rifu fault, NE Japan, Earth Planets Space, 54, this issue, 1021-1026, 2002.

Vermeer, P. A. and R. de Borst, Non-associated plasticity for soils, concrete and rock, Heron, 29, 1-62, 1984.

White, S. H. and P. G. Bretan, Rheological controls on the geometry of deep faults and the tectonic delamination of the continental crust, Tectonics, $\mathbf{4}$, 303-309, 1985.

Zhu, L., Crustal structure across the San Andreas Fault, Southern California from teleseismic converted waves, Earth Planet. Sci. Lett., 179, 183-190, 2000 .

B. E. Hobbs (e-mail: bruce.hobbs@csiro.au), H. Tanaka, and Y. Iio 